\title{
The Importance of Vaccination Against HPV
}

\author{
Tasneem F. Mohammad* and Craig G. Burkhart
}

University of Toledo College of Medicine, Department of Internal Medicine, Section Dermatology, 2341 Old Stone Ct. Apt. 6, Toledo, $\mathrm{OH} 43614$, USA

\begin{abstract}
The human papillomavirus is a DNA virus that is involved in the pathogenesis of several benign and malignant lesions. Although numerous types of HPV exist, vaccines currently target HPV's 6, 11, 16, and 18, which are the most commonly implicated in verrucae and oral-genital neoplasms. Although the HPV vaccine has been recommended as part of the vaccination schedule in adolescents for several years, widespread inoculation has not yet become common practice. It is imperative that vaccination be more broadly instituted to decrease the incidence of HPV related lesions in both males and females.
\end{abstract}

Keywords: Vaccination, HPV.

Infection with HPV occurs when the basement membrane of epithelial cells becomes exposed by trauma. Once inside the cell, the virus enters a maintenance phase where it maintains a basal amount of replication in undifferentiated cells. As the cell differentiates, the replication mechanism changes, with an increase in the level of viral protein synthesis. Spread of the virus occurs as the cornified layers of the epithelium are shed [1].

Transmission of HPV can occur by both vertical and horizontal means. Although periconceptual transmission has been theorized due to the discovery of HPV DNA in the sperm, vas deferens, and proximal portions of the female genital tract [2]; most sources agree that transmission occurs peripartum through an infected birth canal, amniotic fluid, and skin-to-skin contact. Vertical transmission has been shown to cause similar rates of oral and genital infection [3]. Horizontal transmission occurs primarily through sexual contact, with increased prevalence at the commencement of sexual activity and a decline with age secondary to decreased sexual activity and the development of an immune response against the virus [1].

Although between $70-90 \%$ of people are able to clear the HPV virus within one to two years, persistent infection can lead to both benign and dysplastic lesions [4]. HPV types 6 and 11 are most commonly found in verrucae and other benign lesions, such as respiratory papillomas [2]. On the other hand, persistent infection by HPV 16 and 18 often leads to dysplasia and cancer due to increased genetic instability caused by viral proteins. While cervical cancer is the most commonly known neoplasm associated with HPV infection, oral, head and neck, ano-rectal, penile, and vulvovaginal carcinoma is also caused by HPV.

*Address correspondence to this author at the University of Toledo College of Medicine, Department of Internal Medicine, Section Dermatology, 2341 Old Stone Ct. Apt. 6, Toledo, OH 43614, USA; Tel: (31) 550-8412;

E-mails: tasneem mohammad@rockets.utoledo.edu, tasneem646@gmail.com
Because HPV related disease is so prevalent, with cervical carcinoma being the second most common cancer among women worldwide, vaccination offers an important way to decrease morbidity and mortality. Currently, two vaccines against HPV exist. The first, Gardasil, is a tetravalent vaccine against HPV $6,11,16$, and 18, while Cervarix, a divalent vaccine, is active against types 16 and 18. These vaccines act through the L1 major capsid protein that self-assembles into virus-like particles and creates immunity by inducing antibodies to L1. It is currently recommended for both males and females aged 9-26 as a three shot regimen [4].

Despite the availability of vaccines against HPV, widespread vaccination has not yet occurred due to misunderstandings and barriers to administration. In 2012, the Centers for Disease Control and Prevention reported that approximately 54\% of adolescent females aged 13-17 had received at least one dose of the HPV vaccine. However, only $33 \%$ completed the three dose series. In adolescent males, the vaccination rate is even lower, with only $21 \%$ receiving at least one dose of the vaccine [5]. Compared to administration rates of other vaccines, this is extremely low. In order to increase vaccination rates in adolescents, physicians must make an active effort to educate patients about the HPV virus and its impact, as well as ensuring that patients and their parents are receiving accurate information about the vaccine so that they can make an informed decision. Also, a strong recommendation for vaccination from a physician that patients trust can go a long way in helping parents decide whether to allow administration of the vaccine.

To address concerns that patients and their parents have about vaccination, health care providers must have an understanding about barriers to administration of the HPV vaccine. Many parents feel that by vaccinating against a sexually transmitted infection (STI), they will encourage promiscuity and unsafe sexual practices. However, studies in vaccinated populations show no increase in STI's or risky 
sexual behaviors [6]. It is also important to point out to parents that vertical transmission does occur, and that all of us are born with warts. Stressing the action of the HPV vaccine against cancer as opposed to prevention of an STI may make parents more amenable to vaccination. Other parents may be in denial that their children are at risk. This is especially true about male children, because most parents do not realize that HPV can cause oral, penile, and ano-rectal carcinoma in males [7]. By not vaccinating males, an HPV reservoir is also created, allowing for the spread of virus throughout the population. The biggest concern that parents have about vaccination is safety, especially after flawed research showing an association between the measles vaccine and autism received enormous amounts of media attention. This research has since been discredited, and studies on the safety of the HPV vaccine have yielded a favorable side effect profile [6]. Fever, nausea, injection site pain, dizziness, erythema, swelling, and bruising were the most common adverse effects noted with vaccination. Serious effects, such as deep vein thrombosis, were noted in patients who had prior risk factors [8]. Another reason that patients may not receive vaccination is due to cost. Unlike other countries, the American government does not cover the cost of mass vaccination, and some people may find themselves without the means to pay. With the implementation of the Affordable Care Act, this may no longer be an issue because insurance companies will eventually cover all recommended vaccines.

The effects of mass vaccination can be seen using Australia as a model. Australia instituted free vaccination for females in schools beginning in 2007 and males in 2013. Follow up studies have shown a significant decrease in the rates of genital warts in both females and heterosexual males under 30. Although vaccination of males only began this year, they have been reaping the benefits of having immunized sexual partners. Also, no women who were vaccinated against HPV were found to have genital warts in the study's final year [9]. Effects on cancer have not been documented because the vaccine is relatively new, but we would expect rates of HPV related carcinoma to decrease as the adolescents receiving the vaccine transition into adulthood. The ultimate goal is to induce "herd immunity", which is where the prevalence of HPV in the population, or herd, is so low that even people who are not immunized are protected from infection [6]. The UK also instituted the HPV vaccination as part of their national health program in 2008. Although they initially used the bivalent HPV vaccine, they switched to Gardasil in 2012. However, only young females are covered, so the men who have sex with men population are not benefiting from any herd immunity generated, which is a cause for concern as the incidence of HPV related oropharyngeal cancers in males is on the rise [10].

Despite the major advantage that the HPV vaccine confers, other types of HPV not covered by the virus can still cause disease. Tissues from $95 \%$ of warty lesions show more than one type of HPV present, and over $60 \%$ of cervicovaginal specimens contain multiple HPV genotypes. This may be due to a protective influence of low-risk genotypes against environmental exposure and more high- risk HPV types [11]. Currently, a nine-valent vaccine that adds protection against HPV's 31, 33, 45, 52, and 58 is in development to cover other high-risk types. However, the vaccine is not effective in those who have already been infected [6]. For this reason, lifestyle modifications must also be stressed to decrease the transmission of HPV. The number of sexual partners should be kept to a minimum, and safe sexual practices such as condoms and dental dams should be stressed if not in a stable monogamous relationship. Circumcision should be recommended for males and tobacco use should be discouraged due to being a co-factor in HPV related oral carcinoma [1].

In conclusion, the HPV vaccine is a valuable tool in the prevention of HPV related lesions, some of which cause considerable morbidity and mortality. Not only will health care costs related to treatment of these preventable lesions decrease, but our population will be healthier both physically and mentally. Like other STI's, infection with HPV is associated with depression, anxiety, and causes instability in relationships as questions about infidelity inevitably arise [12]. Despite the availability of vaccination, a large portion of the target population has not been inoculated due to misunderstanding and reluctance on the part of parents. However, it is necessary to stress the importance of vaccination and provide accurate information to the public to increase participation and create herd immunity, thus stopping an entirely preventable disease from affecting such a large portion of our population.

\section{CONFLICT OF INTEREST}

The authors confirm that this article content has no conflict of interest.

\section{ACKNOWLEDGEMENTS}

Declared none.

\section{REFERENCES}

[1] Fernandes JV, Galvao A, Joselio M, Araujo De Medeiros F, Thales A. Biology and natural history of human papillomavirus infection. Open J Clin Trials 2013; 5: 1-12.

[2] Syrjanen S. Current concepts on human papillomavirus infections in children. Author J Compil 2010; 118: 494-509.

[3] Smith EM, Parker MA, Rubenstein LM, Haugen TH, Hamsikova E, Turek LP. Evidence for Vertical Transmission of HPV from Mothers to Infants. Infect Dis Obstetr Gynecol 2010; 28(1): 1-7.

[4] Satyaprakash AK, Tyring SK. Human papillomavirus vaccine: A dermatologic perspective. Ind J Dermatol Venereol Leprol 2010; 76(1): 14-9.

[5] Seppa N. HPV is epidemic which is odd since it is largely preventable. ScienceNews 2013; 183(8): 20.

[6] Teen Vaccination Coverage 2012 National Immunization Survey (NIS) - Teen. Centers for Disease Control and Prevention [webpage on the internet]. 2013 Aug; [cited 2013 August 29]: [about 5 screens]. Available from http://www.cdc.gov/vaccines/w ho/teens/vaccination-coverage.html

[7] Burkhart CG. The endogenous, exogenous, and latent infections with human papillomavirus. Int J Dermatol 2004; 43: 548-49.

[8] Pomfret TC, Gagnon JM Jr, Gilchrist AT. Quadrivalent human papillomavirus (HPV) vaccine: a review of safety, efficacy, and pharmacoeconomics. J Clin Pharmacy Ther 2010; 36: 1-9.

[9] Ali H, Donovan B, Wand H, et al. Genital warts in young Australians five years into national human papillomavirus vaccination programme: national surveillance data. BMJ [serial on the internet]. 2013 Apr; [cited 2013 April 18]; 346: [about 14 screens]. Available from: http://www.bmj.com/content/346/bmj.f2032 
[10] Barton S, O'Mahony C. HPV vaccination-reaping the rewards of the appliance of science. BMJ [serial on the internet] 2013; [cited 2013 April 18]; 346: Available from: http://www.bmj.com/content /346/bmj.f2184
[11] Burkhart CG, Burkhart, CN. If papillomavirus found universally in epidermal tissues, what does that mean if a PCR PAP test is positive? Open Dermatol J 2009; 3: 52-3.

[12] Graziottin A, Serafini A. HPV infection in women, psychosexual impact of genital warts and intraepithelial lesions. Int Soc Sexual Med 2009; 6: 633-45.

(C) Mohammad and Burkhart; Licensee Bentham Open.

This is an open access article licensed under the terms of the Creative Commons Attribution Non-Commercial License (http://creativecommons.org/licenses/ by-nc/3.0/) which permits unrestricted, non-commercial use, distribution and reproduction in any medium, provided the work is properly cited. 